\title{
Wie ist die Situation in Deutschland?
}

Für die chronisch obstruktive Lungenerkrankung (COPD) wird in den nächsten Jahren ein Anstieg der Mortalität prognostiziert. Randomisierte kontrollierte Studien liefern zwar wichtige Daten bezüglich der Erkrankung, aber gerade Zahlen aus einer Real-Life-Population wären hier wichtig. H. Worth et al. haben diese nun für Deutschland geliefert. Resp Med 2016; 111: 64-71

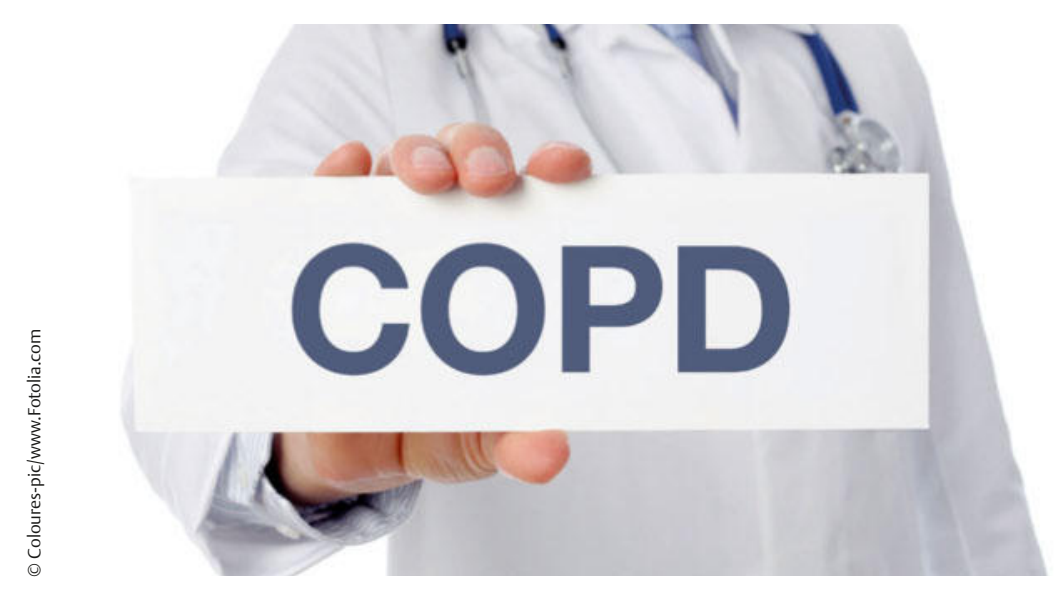

DACCORD (Die ambulante Versorgung mit langwirksamen Bronchodilatatoren: COPD-Register in Deutschland) ist eine longitudinale, prospektive, nicht interventionelle Studie mit dem Ziel, Daten zum Verlauf der COPD unter typischen ambulanten Behandlungsbedingungen zu sammeln. Haupteinschlusskriterien waren die ärztliche Diagnose einer COPD, ein Alter von mindestens 40 Jahren sowie Beginn oder Modifikation einer COPD-Medikation. Die gesammelten Daten beinhalteten u.a. Angaben zur Demographie und Krankheitscharakteristik, zum Raucherstatus, zur Medikation, zu Symptome, zum COPD-Assessment-Test (CAT) und modifizierten Medical Research Council Dyspnoea Score (mMRC), zu Exazerbationen, Begleiterkrankungen und zur forcierten Einsekunden-Kapazität $\left(\mathrm{FEV}_{1}\right)$.

Die 5924 Patienten wurden zwischen November 2012 und November 2013 rekrutiert (59,7\% männlich). Das Durchschnittsalter der Teilnehmer lag bei 65,7 Jahren. 87,1\% von ihnen erhielten eingangs eine COPD-Medikation. Die vorhergesagte $\mathrm{FEV}_{1}$ betrug im Durchschnitt
61,6\%. Zum Beginn der Erhebung berichteten die meisten Patienten Symptome, am häufigsten von Belastungsdyspnoe (85,9\%) und Husten (65,7\%). Gemäß der GOLD-2010-Kriterien wurden 48,6\% der Patienten als GOLD II eingestuft. Bezüglich der GOLD-2011-Klassifikation zeigte das Symptom-Kriterium einen Einfluss: mit dem CAT wurden 43,7 bzw. 45,3\% der Patienten als GOLD B oder D klassifiziert, im mMRC-Score lagen diese Anteile bei 26,4 bzw. 34,0\%. Die Mehrheit der Patienten erhielt ein LAMA-haltiges (Long Acting Muscarinic Antagonist) Therapieregime, insgesamt 39,4\% erhielten inhalative Steroide. Bei 78,3\% der Teilnehmer war mindestens eine Begleiterkrankung bekannt, wobei es sich am häufigsten um kardiovaskuläre Erkrankungen handelte(51,9\%).

\section{Fazit}

DACCORD ist nach Meinung der Autoren eine große prospektive, nicht interventionelle Studie, die ein informatives Bild des typischen COPD-Patienten in Deutschland zeichnet.

Dr. Johannes Weiß, Bad Kissingen 